\title{
Characterization of seminal plasma constituents of unimproved indigenous and Tankwa goats
}

\author{
F.V. Ramukhithi ${ }^{1,2 \#}$, K.A. Nephawe ${ }^{2}$, K.C. Lehloenya ${ }^{3}$, M.M. Seshoka ${ }^{4}$, T. Jonker $^{4}$, A. Kotze ${ }^{5}$, \\ T.C. Chokoe ${ }^{6} \&$ T.L. Nedambale ${ }^{2}$ \\ ${ }^{1}$ Agricultural Research Council, Germplasm Conservation and Reproductive Biotechnologies, Private Bag X 2 , \\ Irene, 0062, South Africa \\ ${ }^{2}$ Tshwane University of Technology, Department of Animal Sciences, Private Bag X 680, Pretoria, 0001, South Africa \\ ${ }^{3}$ University of Zululand, Department of Agriculture, Private Bag X 1001, KwaDlangezwa, 3886, South Africa \\ ${ }^{4}$ Northern Cape Department of Agriculture, Land Reform and Rural Development, Private Bag X 9, Jan Kempdorp, 8550 \\ ${ }^{5}$ National Zoological Gardens of South Africa, P.O. Box 754, Pretoria, 0001, South Africa \\ ${ }^{6}$ Department of Agriculture, Forestry and Fisheries, Directorate: Farm Animal Genetic Resource, Private Bag X 250,
} Pretoria, 0001, South Africa

(Received 16 January 2018; Accepted 22 July 2018; First published online 20 November 2018)

\begin{abstract}
Copyright resides with the authors in terms of the Creative Commons Attribution 4.0 South African License. See: http://creativecommons.org/licenses/by/4.0/za

Condition of use: The user may copy, distribute, transmit and adapt the work, but must recognize the authors and the South African Journal of Animal Science
\end{abstract}

\begin{abstract}
The aim of the study was to characterize and compare seminal plasma constituents of unimproved indigenous and Tankwa goats. In this study, unimproved indigenous (11) and Tankwa (11) goats were used. Their average age was $3 \pm 0.8$ years and average bodyweights were $35.5 \pm 2.1 \mathrm{~kg}$ and $59.8 \pm 1.9 \mathrm{~kg}$, respectively. Semen samples were collected with an electro ejaculator, and evaluated for macroscopic and microscopic semen characteristics and seminal plasma constituents. Both unimproved indigenous and Tankwa goats had normal semen characteristics for goats, with the exception of sperm cell concentration. However, Tankwa goats had higher straight-line velocity and average path velocity compared with unimproved indigenous goats. Unimproved indigenous goats had higher intact acrosome than Tankwa goats. Lipocalin-type prostaglandin-D synthase was one of the constituents that were detected, but it was below the detection limit of $0.5 \mathrm{mmol} / \mathrm{L}$. Moreover, the semen of unimproved indigenous and Tankwa goats had the same levels of fertility-associated antigen, lactate dehydrogenase, sodium, potassium, magnesium, calcium, glucose, cholesterol, triglyceride, lipids and urea. In conclusion, unimproved indigenous and Tankwa goats had the same seminal plasma constituents. These constituents seem to be optimal for the sperm cells, as they resulted in acceptable semen characteristics, except for sperm cell concentration, which was low. Moreover, acceptable standards need to be set for all seminal plasma constituents, as they are not currently known.
\end{abstract}

Keywords: Fertility-associated minerals, genetic variation, semen characteristics

\# Corresponding author: ramukhithif@arc.agric.za

\section{Introduction}

South African indigenous goats are hardy, and tolerant of parasites and diseases. However, some are poorly managed, and under threat of extinction and information about their seminal plasma constituents is limited (Webb et al., 1998; Kotze et al., 2014). For conservation and improvement of indigenous animals, characterization can be a first step before their genetic material is used (Yami \& Merkel, 2008; Yakubu et al., 2010). Animal characterization provides information on the reproductive performance and identification of unique characteristics within populations (Yami \& Merkel, 2008). In South Africa, there is limited information about goat reproductive characteristics (Webb et al., 1998; Matshaba, 2010; Kotze et al., 2014). Therefore, a study aimed at collecting reproductive information from various indigenous goats is important in assisting with future breeding plans and maintaining the unique characteristics of these breeds (Manzi et al., 2011).

Studying seminal plasma constituents can be another method of evaluating the functionality and semen quality of the reproductive system in goats (Teixeira et al., 2009). In other species, such as cattle, birds and fish, the evaluation of seminal plasma constituents such as proteins, sodium, potassium, 
magnesium, calcium, zinc, glucose, cholesterol, triglyceride, lipids and urea has been found useful in the functioning of the sperm cells (Dawson et al., 2002; Massanyi et al., 2008; Bozkurt et al., 2011). Seminal plasma constituents play a major role in sperm cell metabolic processes (Kasimanickam, 2011). However, in small stock, the evaluation of seminal plasma is still not well defined (Gundouan, 2006).

Seminal plasma such as proteins improves buffering capacity and semen quality, triglycerides increase membrane fluidity, and glucose provides osmotic balance and energy to the sperm cells (Purdy, 2006). Urea and zinc are responsible for sperm cell development (Cortada et al., 2000; Massanyi et al., 2008). Cholesterol is responsible for sperm cell capacitation (Cross, 1998). Lipids are responsible for maintaining sperm cell membrane structure, and for sperm cell metabolism, capacitation and fertilization (Gundouan, 2006). Calcium is required for sperm cell physiology, motility and acrosome reaction (Sorensen et al., 1999). Sodium improves sperm cell motility (Massanyi et al., 2008). In addition, magnesium is required for the enzymes that act on substrates containing phosphate (Nikoobakht et al., 2005). Potassium is responsible for sperm cell volume regulation (Klein et al., 2006). This function is necessitated when the sperm cells encounter a hypo-osmotic challenge on ejaculation into the female tract (Yeung \& Cooper, 2008).

However, in goats, information about the seminal plasma constituents that are vital to the functioning of sperm cells is limited. Therefore, the objective of this study was to characterize and compare the seminal plasma constituents of unimproved indigenous and Tankwa goats.

\section{Materials and Methods}

The study was approved by the Ethics Committee of the Agricultural Research Council (ARC) (APIEC15/044) and Tshwane University of Technology (REC2012/10/019-2). In the current study, 11 unimproved indigenous bucks from the ARC and 11 Tankwa bucks from Carnarvon Research Station were used. Unimproved indigenous goats are mostly small animals, which tolerate harsh environmental conditions, parasites and diseases, have good maternal ability and are able to survive on poor-quality grazing compared with exotic breeds (Ramsay \& Donkin, 2000). Tankwa goats were first discovered in the Tankwa Karoo National Park in the Northern Cape. It is thought that this type of goat represents former indigenous goats from the area that date back to the 1900s. Since then, the goats have been separated from other goat breeds in the area owing to fencing of the park. As a result, these goats look distinct from other breeds (Jonker, 2013; Kotze et al., 2014). The average age of the bucks used in the current study was $3 \pm$ 0.8 years (matured). The average bodyweight for unimproved indigenous bucks was $35.5 \pm 2.1 \mathrm{~kg}$ and for Tankwa bucks was $59.8 \pm 1.9 \mathrm{~kg}$. All the bucks were grazing on natural pasture and had access to drinking water ad libitum.

Prior to semen collection with an electro ejaculator, hair around the sheath was shaved with scissors and the prepuce was cleaned with a paper towel containing $70 \%$ ethanol. The rectal probe was washed with $70 \%$ ethanol and lubricated with a jelly. The rectal probe was inserted and placed in the rectum above the accessory sex glands. Semen samples were collected in pre-warmed $\left(37^{\circ} \mathrm{C}\right) 15 \mathrm{~mL}$ graduated tubes. Four levels of 30 voltage $(\mathrm{V})$ were applied when the bucks were lying on their side. Following the collection of semen, samples were evaluated for volume by reading the measurements on the collection tubes. Semen $\mathrm{pH}$ was evaluated with a $\mathrm{pH}$ meter (HANNA Instruments ${ }^{\circledR}$, South Africa), whereby an electrode was rinsed with sterile water and wiped with a paper towel before being placed for 30 seconds in a tube that contained the semen sample.

Sperm cell motility was determined objectively using a Sperm Class Analyser ${ }^{\circledR}$ (Microptic S.L, Barcelona). The amount of $500 \mu \mathrm{L}$ Ham's F-10 (Sigma-Aldrich, South Africa) and $10 \mu \mathrm{L}$ semen were mixed in a $1 \mathrm{~mL}$ graduated tube and incubated for 5 minutes at $37^{\circ} \mathrm{C}$. Following incubation, $10 \mu \mathrm{L}$ extended semen was placed on a pre-warmed microscopic slide $\left(37^{\circ} \mathrm{C}\right)$, mounted with a cover slip, and examined (x 10) under a phase contrast microscope. Sperm cell motility was categorized as progression [total motility (TM); progressive motility (PM) and non-progressive motility (NPM)], velocity [rapid (81-180 $\mu \mathrm{m} / \mathrm{s})$, medium (51-80 $\mu \mathrm{m} / \mathrm{s})$ and slow $(\leq 50 \mu \mathrm{m} / \mathrm{s})$ ] and average values of velocity parameters [curvilinear velocity (VCL) measures a sperm cell movement along its actual path; straight-line velocity (VSL) measures a sperm cell movement along a straight line; average path velocity (VAP) measures smoothed cell path; linearity (LIN) = VSL/VCL; straightness $($ STR $)=$ VSL/VAP and wobble $($ WOB $)=$ VSL/VAP]. Sperm cell concentration was determined with a spectrophotometer (Jenway, United Kingdom). A square cuvette was filled with $3 \mathrm{~mL}$ of sodium citrate solution and placed in a spectrophotometer (Jenway, United Kingdom) for at least 30 seconds. A semen sample $(15 \mu \mathrm{L})$ was added in a square cuvette containing the sodium citrate solution, again placed in a spectrophotometer (Jenway, United Kingdom) to read the absorbance. The absorbance value was used to determine the final sperm cell concentration with the aid of the formula ( $201 \times 25.97 \times$ absorbance -0.3$)$.

To evaluate acrosome integrity, sperm cell viability, morphology and abnormalities, samples stained with nigrosin-eosin stain were examined under a fluorescent microscope (Olympus, Japan) and 200 sperm 
cells per slide were counted. Live sperm cells and the sperm cells that had non-damaged acrosomes did not absorb stain (fluorescence), while dead sperm cells and sperm cells that had damaged acrosomes absorbed stain and became purple in colour (Samper, 2000). Live sperm cells were further evaluated for morphology and abnormalities. Abnormalities were categorized as primary, secondary and tertiary, as classified by Loskutoff \& Crichton (2001). To evaluate membrane integrity, a hypo-osmotic swelling test was used. The samples were evaluated under a phase contrast microscope $(400 \mathrm{x})$ and 200 sperm cells per slide were counted. Sperm cells with swollen and coiled tails were considered intact (Naing et al., 2010).

Following semen evaluation, seminal plasma was collected by centrifugation (Naing et al., 2011) at $1500 \mathrm{rpm}$ for 5 minutes and was stored at $-20{ }^{\circ} \mathrm{C}$ until analysis. Because of high costs of determining seminal plasma constituents, eleven samples per breed were evaluated. Determination of seminal plasma constituents was done using commercial kits at Carnatlab South Africa (Pty) Ltd. To determine sodium, potassium, magnesium, zinc and calcium, seminal plasma was centrifuged at $1500 \mathrm{rpm}\left(4^{\circ} \mathrm{C}\right)$ for 20 minutes. Then $1 \mathrm{M}$ sodium hydroxide was added to form a coloured salt complex. The concentration elements in the coloured complex solution were determined spectrophotometrically (fluorescent colour intensity) using a digital fluorescent microscope.

Determination of osteopontin, metalloproteinases type-2 tissue inhibitor, fertility-associated antigen and urea was carried out by adding $100 \mu \mathrm{L}$ standards and sample solution to the appropriate wells, shaken gently to mix the contents, and then incubated at $37^{\circ} \mathrm{C}$ for 1 hour. The wells were washed seven times with $400 \mu \mathrm{L}$ of wash solution. After the final wash, the wells were tapped firmly on a lint-free paper towel to remove any remaining wash buffer. A labelled antibody $(100 \mu \mathrm{L})$ was pipetted into each well. The plates were sealed and incubated at $4{ }^{\circ} \mathrm{C}$ for 30 minutes. The plates were emptied of their contents and washed nine times with $400 \mu \mathrm{L}$ wash solution. After the final wash, the plates were emptied and the results were generated by adding $100 \mu \mathrm{L}$ of tetramethylbenzidine substrate to each well. The plates were incubated for 30 minutes at $25{ }^{\circ} \mathrm{C}$ in the dark. The reaction was halted with $100 \mu \mathrm{L}$ stop solution and the absorbance read at $450 \mathrm{~nm}$.

Lactose dehydrogenase was determined by the catalytic oxidation of lactate to pyruvate in the presence of nicotinamide adenine dinucleotide (NAD) with subsequent reduction to nicotinamide adenine dinucleotide hydrogen (NADH). The rate of $\mathrm{NADH}$ formation measured at $340 \mathrm{~nm}$ was directly proportional to serum lactate dehydrogenase activity. The change in absorbance measured at $340 \mathrm{~nm}$ was directly proportional to the activity of lactate dehydrogenase in the sample, and translated to the concentration of lactose dehydrogenase in the sample. Lactate dehydrogenase reagent $1(4 \times 40 \mathrm{~mL})$ and lactate dehydrogenase reagent $2(4 \times 8 \mathrm{~mL})$ were reacted with the sample. The enzyme activity was measured spectrophotometrically (fluorescent colour intensity) (lactic acid + NAD $+\rightarrow$ pyruvate + NADH).

Triglycerides were determined with a spectrophotometer, which was set at a wavelength of $540 \mathrm{~nm}$, and the absorbance reading to zero with water was used as a reference. Free glycerol and triglycerides reagents were prepared and warmed up to assay temperature in the blank, standard and sample cuvettes. Free glycerol and triglyceride reagents $(0.8 \mathrm{~mL})$ were pipetted into each cuvette. Then $10 \mathrm{~mL}$ of water, glycerol standard and sample were added to the blank, standard and sample cuvettes and mixed with gentle inversion. The cuvettes were then incubated for 5 minutes at $37^{\circ} \mathrm{C}$. Initial absorbance reading of blank, standard and sample was taken at $540 \mathrm{~nm}$ versus water as the reference. To each cuvette, $0.2 \mathrm{~mL}$ reconstituted triglycerides reagent was added, mixed and incubated for 5 minutes at $37{ }^{\circ} \mathrm{C}$. The final absorbance of the cuvettes was read at $540 \mathrm{~nm}$ versus water as the reference and the triglycerides concentration was calculated.

Lipocalin-type prostaglandin D synthase was determined using a spectrophotometer. Seminal plasma was incubated overnight $(12 \mathrm{~h})$ at $48{ }^{\circ} \mathrm{C}$ with $30 \mathrm{mg}$ of anti-recombinant bovine lipocalin-type PGD synthase IgG. Samples were washed three times with PBS-BSA and re-suspended to $500 \mathrm{~mL}$ FC blocking medium. Five microlitre $(0.5 \mathrm{mg} / \mathrm{mL})$ of fluorescein isothiocyanate-conjugated goat anti-rabbit lgG Fab fragment was added to $500 \mathrm{~mL}$ seminal plasma suspension for 1 hour. After being washed three times in PBS-BSA as before, the fluorescence of the samples (concentrations) was measured using a digital fluorescent microscopy. For lipid determination, seminal plasma lipids were extracted with six volumes of chloroformmethanol $(2 / 1, V / V)$, centrifuged at $800 \times \mathrm{g}$ for 3 minutes. The resulting lower phase was aspirated and dried under a stream of nitrogen. Total lipids were determined enzymatically and their quantities were measured colorimetrically. Seminal plasma constituents and semen characteristics data were analysed using the generalised linear model (GLM) procedures of Statistical Analysis Software (SAS), version 9.2 (1999). Least square means (LSM) were compared using the least significance difference (LSD) test $(\alpha=0.05)$. The following statistical model was used: 


$$
y_{i j}=\mu+T_{i}+e_{i j}
$$

where: $y_{i j}$ is the observation of the $j$-th experimental unit of the $\mathrm{i}$-th treatment $\mu$ is the overall mean; $T_{i}$ is the effect of the $i$-th treatment or breed $\mathrm{e}_{\mathrm{ij}}$ is the residual (error) component.

The Pearson correlation was done to show the relationship between semen parameters and seminal plasma constituents.

\section{Results}

The seminal plasma constituents of unimproved indigenous and Tankwa goats are indicated in Table 1. Lipocalin-type prostaglandin $D$ synthase content was below the detection limit of $0.5 \mathrm{mmol} / \mathrm{L}$ and osteopontin quantity could not be measured. Metalloproteinase type-2 tissue inhibitor and zinc were not detected. For all the seminal plasma constituents that were detected, no difference $(P>0.05)$ observed between the unimproved indigenous and Tankwa goats.

Table 1 Seminal plasma constituents of unimproved indigenous and Tankwa goats

\begin{tabular}{lcc}
\hline Seminal plasma constituents & Breed & Tankwa (n = 11) \\
\hline & Unimproved $(\mathbf{n}=\mathbf{1 1})$ & Positive \\
Osteopontin & Positive & $<0.5 \pm 0$ \\
Lipocalin-type prostaglandin D synthase $(\mathrm{mmol} / \mathrm{L})$ & $<0.5 \pm 0$ & $0.13 \pm 0.02$ \\
Fertility associated antigen $(\mathrm{mg} / \mathrm{mL})$ & $0.13 \pm 0.02$ & $\mathrm{ND}$ \\
Metalloproteinases type-2 tissue inhibitor $(\mathrm{mmol} / \mathrm{L})$ & $\mathrm{ND}$ & $277.8 \pm 43.0$ \\
Lactate dehydrogenase $(\mathrm{IU} / \mathrm{L})$ & $251.0 \pm 41.5$ & $569.2 \pm 22.1$ \\
Sodium $(\mu \mathrm{g} / \mathrm{mL})$ & $590.4 \pm 15.6$ & $277.7 \pm 11.7$ \\
Potassium $(\mu \mathrm{g} / \mathrm{mL})$ & $269.4 \pm 9.9$ & $7.7 \pm 0.4$ \\
Magnesium $(\mu \mathrm{g} / \mathrm{mL})$ & $6.6 \pm 0.3$ & $4.1 \pm 0.3$ \\
Calcium $(\mu \mathrm{g} / \mathrm{mL})$ & $4.1 \pm 0.2$ & $\mathrm{ND}$ \\
Zinc $(\mathrm{mmol} / \mathrm{L})$ & $\mathrm{ND}$ & $49.1 \pm 2.0$ \\
Glucose $(\mathrm{mg} / \mathrm{mL})$ & $47.3 \pm 1.8$ & $1.1 \pm 0.4$ \\
Cholesterol $(\mathrm{mmol} / \mathrm{L})$ & $1.1 \pm 0.1$ & $0.2 \pm 0.01$ \\
Triglyceride $(\mathrm{mmol} / \mathrm{L})$ & $0.1 \pm 0.01$ & $2.5 \pm 0.09$ \\
Lipids $(\mathrm{mmol} / \mathrm{L})$ & $2.4 \pm 0.06$ & $50.5 \pm 1.8$ \\
Urea $(\mu \mathrm{gg} / \mathrm{mL})$ & $49.8 \pm 1.4$ & \\
\hline
\end{tabular}

$\overline{\mathrm{a}, \mathrm{b}}$ Values with different superscripts within the same row differ significantly $(P<0.05)$, ND: not detected

Semen characteristics of unimproved indigenous and Tankwa goats evaluated for seminal plasma constituents are depicted in Table 2. There were differences $(P<0.05)$ between unimproved indigenous and Tankwa goats in VSL $(53.2 \pm 15.1 \mathrm{vs} 99.2 \pm 5.6 \mu \mathrm{m} / \mathrm{s}$, respectively), VAP $(71.4 \pm 17.4 \mathrm{vs} 124.7 \pm 5.3 \mu \mathrm{m} / \mathrm{s}$, respectively) and intact acrosome (95.4 \pm 2.2 vs $77.2 \pm 2.8 \%$, respectively). However, means for sperm cell concentration, progression, viability, morphology and abnormalities, VCL, linearity, STR, wobble, velocity parameters, membrane integrity, semen volume and $\mathrm{pH}$ of unimproved indigenous and Tankwa goat were not different $(P>0.05)$.

The correlations between seminal plasma constituents and semen parameters of unimproved indigenous goats are presented in Table 3 . None of the seminal plasma constituents had a correlation with semen $\mathrm{pH}$. Potassium had a negative correlation with VAP $(r=-0.681, P<0.05)$, semen volume, sperm cell concentration, TM, NPM, medium velocity, VCL, intact acrosome, non-intact membrane, dead and abnormal sperm cells, primary and secondary abnormalities $(P>0.05)$. Magnesium had a positive correlation with primary abnormalities $(r=0.700, P<0.05)$. Calcium had a positive correlation with tertiary abnormalities $(r=0.753, P$ $<0.05)$. However, it had a negative correlation with primary abnormalities $(r=0.632, P<0.05)$. Cholesterol had a 
negative correlation with rapid velocity $(r=-0.642)$, VCL $(r=-0.717)$ and VSL $(r=-0.699)(P<0.05)$. Triglycerides had a positive correlation with non-intact membrane $(r=-0.723)$ and dead sperm cells $(r=-0.734)$ $(P<0.05)$. However, it had a negative correlation with rapid velocity $(r=-0.284)(P<0.05)$. Lipids had a positive correlation with tertiary abnormalities $(r=0.688, P<0.05)$.

Table 2 Semen characteristics of unimproved indigenous and Tankwa goats

\begin{tabular}{|c|c|c|c|c|c|c|}
\hline \multirow{2}{*}{\multicolumn{5}{|c|}{ Characteristics }} & \multicolumn{2}{|c|}{ Breed } \\
\hline & & & & & Unimproved $(n=11)$ & Tankwa $(n=11)$ \\
\hline \multirow{2}{*}{ Semen } & & & \multirow{2}{*}{\multicolumn{2}{|c|}{$\begin{array}{l}\text { Volume }(\mathrm{mL}) \\
\mathrm{pH}\end{array}$}} & $0.7 \pm 0.12$ & $0.9 \pm 0.03$ \\
\hline & & & & & $7 \pm 0.1$ & $7 \pm 0.1$ \\
\hline & \multicolumn{4}{|c|}{ Concentration (X 109/mL) } & $1.8 \pm 0.21$ & $1.12 \pm 0.17$ \\
\hline & & \multirow{4}{*}{ Progression (\%) } & \multicolumn{2}{|l|}{ TM } & $78.2 \pm 8.5$ & $75.0 \pm 6.1$ \\
\hline & & & \multicolumn{2}{|l|}{ PM } & $35.4 \pm 7.7$ & $44.4 \pm 6.3$ \\
\hline & & & \multicolumn{2}{|l|}{ NPM } & $42.8 \pm 7.6$ & $30.5 \pm 3.1$ \\
\hline & & & \multicolumn{2}{|l|}{ Static } & $21.8 \pm 8.5$ & $25.0 \pm 6.1$ \\
\hline & & & \multicolumn{2}{|l|}{ Rapid } & $45.6 \pm 7.2$ & $41.2 \pm 5.9$ \\
\hline & & Velocity (\%) & \multicolumn{2}{|l|}{ Medium } & $26.8 \pm 8.2$ & $29.9 \pm 4.5$ \\
\hline & & & \multicolumn{2}{|l|}{ Slow } & $5.8 \pm 1.3$ & $7.7 \pm 1.0$ \\
\hline & & & \multicolumn{2}{|l|}{$\mathrm{VCL}(\mu \mathrm{m} / \mathrm{s})$} & $101.7 \pm 18.9$ & $186.0 \pm 8.4$ \\
\hline & Motility & & \multicolumn{2}{|l|}{ VSL $(\mu \mathrm{m} / \mathrm{s})$} & $53.2^{a} \pm 15.1$ & $99.2^{b} \pm 5.6$ \\
\hline & & Average values of & \multicolumn{2}{|l|}{$\operatorname{VAP}(\mu \mathrm{m} / \mathrm{s})$} & $71.4^{a} \pm 17.4$ & $124.7^{b} \pm 5.3$ \\
\hline & & parameters & \multicolumn{2}{|c|}{ Linearity (\%) } & $49.8 \pm 6.1$ & $53.5 \pm 2.2$ \\
\hline & & & \multicolumn{2}{|c|}{ Straightness (\%) } & $72.3 \pm 4.7$ & $79.3 \pm 1.9$ \\
\hline & & & \multicolumn{2}{|l|}{ Wobble (\%) } & $66.9 \pm 5.1$ & $67.2 \pm 1.5$ \\
\hline \multirow{11}{*}{$\begin{array}{l}\text { Sperm } \\
\text { cell }\end{array}$} & \multirow{4}{*}{\multicolumn{2}{|c|}{ Vitality (\%) }} & \multirow{2}{*}{ Acrosome } & Intact & $95.4^{a} \pm 2.2$ & $77.2^{b} \pm 2.8$ \\
\hline & & & & Non-intact & $4.6^{a} \pm 2.2$ & $22.8^{b} \pm 2.8$ \\
\hline & & & \multirow{2}{*}{ Membrane } & Intact & $82.1 \pm 2.3$ & $74.0 \pm 2.3$ \\
\hline & & & & Non-intact & $17.9 \pm 2.3$ & $26.0 \pm 2.3$ \\
\hline & \multirow{2}{*}{\multicolumn{2}{|c|}{ Viability (\%) }} & Live & & $83.5 \pm 3.6$ & $76.0 \pm 2.0$ \\
\hline & & & Dead & & $16.5 \pm 3.6$ & $24.0 \pm 2.0$ \\
\hline & \multirow{2}{*}{\multicolumn{2}{|c|}{ Morphology (\%) }} & Normal & & $92.0 \pm 1.8$ & $93.7 \pm 1.7$ \\
\hline & & & Abnormal & & $9.0 \pm 1.5$ & $7.0 \pm 1.7$ \\
\hline & \multirow{3}{*}{\multicolumn{2}{|c|}{ Abnormalities (\%) }} & Primary & & $1.4 \pm 0.3$ & $1.2 \pm 0.7$ \\
\hline & & & Secondary & & $4.3 \pm 1.1$ & $4.3 \pm 0.6$ \\
\hline & & & Tertiary & & $1.6 \pm 0.4$ & $0.8 \pm 0.4$ \\
\hline
\end{tabular}

${ }^{a, b}$ Values with different superscripts within the same row differ significantly $(P<0.05)$

TM: total motility, PM: progressive motility, NPM: non progressive motility, VCL: curvilinear velocity, VSL: straight-line velocity, VAP: average path velocity

The correlations between the seminal plasma constituents of Tankwa goats and sperm cell characteristics are presented in Table 4 . None of the seminal plasma constituents had a correlation with semen $\mathrm{pH}$. Fertility-associated antigen had a negative correlation with slow $(r=-0.818)$ and medium velocity $(r=-0.851)(P<0.01)$. 
Table 3 Pearson correlation coefficients between seminal plasma constituents and semen parameters of unimproved indigenous goats

\begin{tabular}{|c|c|c|c|c|c|c|c|c|c|c|c|c|c|}
\hline \multirow{2}{*}{\multicolumn{3}{|c|}{ Semen parameters }} & \multicolumn{11}{|c|}{ Seminal plasma constituents } \\
\hline & & & \multirow{2}{*}{$\begin{array}{l}\text { FAA } \\
0.122\end{array}$} & \multirow{2}{*}{$\begin{array}{c}\text { LDH } \\
-0.629\end{array}$} & \multirow{2}{*}{$\begin{array}{c}\mathrm{Na} \\
0.227\end{array}$} & \multirow{2}{*}{$\begin{array}{c}\mathbf{K} \\
-0.179\end{array}$} & \multirow{2}{*}{$\begin{array}{c}\mathbf{M g} \\
0.396\end{array}$} & \multirow{2}{*}{$\frac{\mathrm{Ca}}{-0.064}$} & \multirow{2}{*}{$\begin{array}{c}\text { Glu } \\
0.009\end{array}$} & \multirow{2}{*}{$\begin{array}{c}\text { Cho } \\
-0.445\end{array}$} & \multirow{2}{*}{$\begin{array}{c}\text { Tri } \\
-0.235\end{array}$} & \multirow{3}{*}{$\begin{array}{c}\text { Lipids } \\
\begin{array}{c}-0.350 \\
0\end{array}\end{array}$} & \multirow{2}{*}{$\begin{array}{r}\text { Urea } \\
-0.358\end{array}$} \\
\hline & & Volume & & & & & & & & & & & \\
\hline semen & & $\mathrm{pH}$ & 0 & 0 & 0 & 0 & 0 & 0 & 0 & 0 & 0 & & 0 \\
\hline \multirow{25}{*}{$\begin{array}{l}\text { Sperm } \\
\text { cell }\end{array}$} & Concentration & $(\mathrm{X} \mathrm{10} / \mathrm{mL})$ & 0.328 & -0.527 & 0.167 & -0.153 & 0.361 & -0.193 & -0.578 & -0.482 & -0.396 & -0.370 & -0.530 \\
\hline & \multirow{4}{*}{ Progression (\%) } & TM & 0.330 & 0.282 & 0.081 & -0.101 & -0.116 & 0.197 & 0.179 & -0.603 & -0.135 & 0.536 & -0.600 \\
\hline & & PM & -0.024 & -0.153 & -0.581 & 0.531 & -0.140 & -0.095 & 0.376 & -0.534 & -0.506 & 0.330 & -0.211 \\
\hline & & NPM & 0.292 & 0.349 & 0.504 & -0.483 & 0.008 & 0.235 & -0.134 & -0.098 & 0.267 & 0.196 & -0.338 \\
\hline & & Static & -0.330 & -0.282 & -0.081 & 0.101 & 0.116 & -0.197 & -0.179 & 0.603 & 0.135 & -0.536 & 0.600 \\
\hline & \multirow{3}{*}{ Velocity (\%) } & Rapid & 0.177 & 0.214 & -0.448 & 0.113 & -0.170 & 0.206 & 0.426 & $-0.642^{\star}$ & $-0.284^{*}$ & 0.533 & -0.442 \\
\hline & & Medium & 0.234 & 0.073 & 0.558 & -0.232 & 0.112 & -0.044 & -0.225 & -0.078 & 0.146 & 0.029 & -0.209 \\
\hline & & Slow & -0.291 & 0.186 & -0.507 & 0.173 & -0.507 & 0.414 & 0.235 & 0.094 & -0.236 & 0.349 & -0.139 \\
\hline & \multirow{6}{*}{$\begin{array}{l}\text { Average values of } \\
\text { velocity } \\
\text { parameters }\end{array}$} & $\mathrm{VCL}(\mu \mathrm{m} / \mathrm{s})$ & 0.407 & 0.221 & -0.198 & -0.213 & 0.248 & 0.059 & 0.060 & $-0.717^{*}$ & -0.153 & 0.296 & -0.449 \\
\hline & & VSL $(\mu \mathrm{m} / \mathrm{s})$ & 0.258 & 0.066 & -0.370 & 0.063 & 0.276 & -0.087 & 0.050 & $-0.699 *$ & -0.333 & 0.222 & -0.280 \\
\hline & & $\operatorname{VAP}(\mu \mathrm{m} / \mathrm{s})$ & 0.227 & -0.025 & 0.039 & $-0.681^{*}$ & -0.265 & 0.256 & -0.059 & -0.167 & -0.322 & -0.521 & 0.226 \\
\hline & & LIN (\%) & -0.193 & -0.100 & -0.365 & 0.541 & 0.057 & -0.178 & 0.039 & -0.318 & -0.469 & 0.164 & 0.109 \\
\hline & & STR (\%) & -0.380 & -0.266 & -0.485 & 0.516 & 0.079 & -0.205 & -0.028 & -0.030 & -0.308 & -0.054 & 0.310 \\
\hline & & WOB (\%) & -0.017 & 0.061 & -0.254 & 0.493 & -0.010 & -0.145 & 0.080 & -0.402 & -0.508 & 0.259 & 0.008 \\
\hline & \multirow{4}{*}{ Vitality (\%) } & A/intact & -0.311 & -0.167 & 0.362 & -0.007 & -0.374 & 0.178 & 0.060 & 0.623 & 0.204 & -0.177 & 0.154 \\
\hline & & A/non-intact & 0.311 & 0.167 & -0.362 & 0.007 & 0.374 & -0.178 & -0.060 & -0.623 & -0.204 & 0.177 & -0.154 \\
\hline & & M/intact & 0.227 & -0.322 & -0.360 & 0.444 & 0.114 & -0.385 & -0.345 & -0.547 & $-0.723^{\star}$ & 0.019 & -0.381 \\
\hline & & M/non-intact & -0.227 & 0.322 & 0.360 & -0.444 & -0.114 & 0.385 & 0.345 & 0.547 & $0.723^{\star}$ & -0.019 & 0.381 \\
\hline & \multirow{2}{*}{ Viability (\%) } & Live & 0.131 & -0.521 & -0.246 & 0.621 & 0.029 & -0.484 & -0.280 & -0.262 & $-0.734^{\star}$ & -0.371 & 0.025 \\
\hline & & Dead & -0.131 & 0.521 & 0.246 & -0.621 & -0.029 & 0.484 & 0.280 & 0.262 & $0.734^{\star}$ & 0.371 & -0.025 \\
\hline & \multirow{2}{*}{ Morphology (\%) } & Normal & -0.028 & -0.226 & -0.093 & 0.386 & -0.019 & -0.258 & 0.243 & 0.017 & -0.199 & -0.215 & 0.322 \\
\hline & & Abnormal & 0.028 & 0.226 & 0.093 & -0.386 & 0.019 & 0.258 & -0.243 & -0.017 & 0.199 & 0.215 & -0.322 \\
\hline & \multirow{3}{*}{ Abnormalities (\%) } & Primary & 0.462 & -0.294 & 0.340 & -0.050 & $0.700^{*}$ & $-0.632^{\star}$ & -0.126 & -0.032 & -0.565 & -0.609 & 0.127 \\
\hline & & Secondary & 0.229 & -0.007 & -0.100 & -0.207 & 0.081 & -0.050 & -0.305 & -0.042 & -0.059 & -0.070 & -0.276 \\
\hline & & Tertiary & -0.546 & 0.283 & -0.056 & 0.012 & -0.564 & $0.753^{\star}$ & -0.134 & -0.154 & 0.252 & $0.688^{\star}$ & -0.337 \\
\hline
\end{tabular}

*Significant at $P<0.05$. FAA: fertility associated antigen, LDH: lactate dehydrogenase, Na: sodium, K: potassium, Mg: magnesium, Ca: calcium, Glu: glucose, A: acrosome, M: membrane, Cho: cholesterol, Tri: triglyceride, TM: total motility, PM: progressive motility, NPM: non-progressive motility, VCL: curvilinear velocity, VSL: straight-line velocity, VAP: average path velocity, LIN: linearity, STR: straightness, WOB: wobble 
Table 4 Pearson correlation coefficients between seminal plasma constituents and semen parameters of Tankwa goats

\begin{tabular}{|c|c|c|c|c|c|c|c|c|c|c|c|c|c|}
\hline \multicolumn{3}{|c|}{ Semen parameters } & \multicolumn{11}{|c|}{ Seminal plasma constituents } \\
\hline & & & FAA & LDH & $\mathrm{Na}$ & $\mathbf{K}$ & Mg & $\mathrm{Ca}$ & Glu & Cho & Tri & Lipids & Urea \\
\hline \multirow{2}{*}{\multicolumn{2}{|c|}{ Semen }} & Volume & -0.395 & 0.026 & -0.159 & 0.096 & 0.611 & $0.663^{\star}$ & 0.411 & -0.433 & -0.035 & 0.480 & -0.231 \\
\hline & & $\mathrm{pH}$ & 0 & 0 & 0 & 0 & 0 & 0 & 0 & 0 & 0 & 0 & 0 \\
\hline \multirow{25}{*}{$\begin{array}{l}\text { Sperm } \\
\text { cell }\end{array}$} & Concentration & $\left(X 10^{9} / \mathrm{mL}\right)$ & -0.477 & -0.063 & 0.337 & -0.232 & 0.247 & 0.220 & 0.391 & 0.204 & -0.579 & 0.222 & -0.250 \\
\hline & \multirow{4}{*}{ Progression (\%) } & TM & -0.439 & 0.287 & 0.192 & 0.166 & -0.150 & 0.644 & -0.137 & -0.529 & -0.166 & -0.130 & -0.147 \\
\hline & & PM & -0.489 & 0.219 & 0.294 & 0.360 & -0.079 & 0.358 & -0.247 & -0.494 & -0.481 & -0.025 & 0.012 \\
\hline & & NPM & 0.130 & 0.113 & -0.210 & -0.395 & -0.127 & 0.519 & 0.226 & -0.031 & $0.634^{\star}$ & -0.198 & -0.305 \\
\hline & & Static & 0.439 & -0.287 & -0.192 & -0.166 & 0.150 & $-0.645^{\star}$ & 0.137 & 0.529 & 0.166 & 0.130 & 0.147 \\
\hline & \multirow{3}{*}{ Velocity (\%) } & Rapid & 0.276 & $0.686^{\star}$ & -0.264 & 0.198 & -0.372 & 0.072 & -0.109 & -0.302 & 0.016 & -0.266 & -0.008 \\
\hline & & Medium & -0.851 ** & -0.499 & 0.065 & 0.102 & 0.303 & 0.592 & 0.241 & -0.048 & -0.377 & 0.030 & 0.190 \\
\hline & & Slow & $-0.818^{\star \star}$ & -0.179 & 0.189 & -0.021 & 0.003 & $0.639^{\star}$ & 0.163 & -0.130 & -0.440 & 0.028 & -0.086 \\
\hline & \multirow{6}{*}{$\begin{array}{l}\text { Average values of } \\
\text { velocity parameters }\end{array}$} & $\mathrm{VCL}(\mu \mathrm{m} / \mathrm{s})$ & 0.126 & $0.723^{\star \star}$ & 0.231 & 0.124 & -0.315 & 0.339 & -0.305 & -0.297 & 0.071 & -0.322 & -0.275 \\
\hline & & VSL $(\mu \mathrm{m} / \mathrm{s})$ & -0.080 & 0.537 & -0.016 & 0.513 & -0.197 & 0.146 & 0.252 & -0.324 & -0.333 & -0.247 & 0.178 \\
\hline & & $\operatorname{VAP}(\mu \mathrm{m} / \mathrm{s})$ & 0.061 & 0.608 & 0.054 & 0.344 & -0.242 & 0.340 & -0.234 & -0.309 & -0.166 & -0.371 & 0.023 \\
\hline & & LIN (\%) & -0.315 & -0.071 & -0.327 & 0.602 & 0.083 & -0.066 & 0.036 & -0.122 & -0.518 & -0.003 & 0.570 \\
\hline & & STR (\%) & -0.025 & -0.156 & -0.117 & -0.274 & -0.489 & -0.156 & -0.117 & -0.274 & -0.489 & 0.046 & 0.446 \\
\hline & & WOB (\%) & -0.415 & -0.279 & -0.413 & 0.456 & 0.169 & 0.034 & 0.191 & 0.014 & -0.464 & 0.067 & 0.620 \\
\hline & \multirow{4}{*}{ Vitality (\%) } & A/intact & 0.425 & 0.345 & -0.008 & -0.546 & -0.534 & -0.268 & -0.212 & 0.123 & 0.339 & 0.140 & -0.608 \\
\hline & & A/non-intact & -0.425 & -0.345 & 0.008 & 0.546 & 0.534 & 0.268 & 0.212 & -0.123 & -0.339 & -0.140 & 0.608 \\
\hline & & M/intact & 0.527 & 0.175 & 0.216 & 0.067 & 0.361 & -0.187 & 0.435 & -0.021 & 0.223 & 0.232 & -0.080 \\
\hline & & M/non-intact & -0.527 & -0.175 & -0.216 & -0.067 & -0.361 & 0.187 & -0.435 & 0.021 & -0.223 & -0.232 & 0.080 \\
\hline & \multirow{2}{*}{ Viability (\%) } & Live & 0.605 & 0.340 & 0.048 & -0.337 & -0.131 & -0.310 & 0.139 & 0.103 & 0.350 & 0.246 & -0.447 \\
\hline & & Dead & -0.605 & -0.340 & -0.048 & 0.337 & 0.131 & 0.310 & -0.139 & -0.103 & -0.350 & -0.246 & 0.447 \\
\hline & \multirow{2}{*}{ Morphology (\%) } & Normal & 0.345 & -0.232 & 0.025 & -0.239 & 0.060 & -0.349 & -0.141 & 0.533 & 0.233 & -0.525 & 0.259 \\
\hline & & Abnormal & -0.345 & 0.232 & -0.025 & 0.239 & -0.060 & 0.349 & 0.141 & -0.533 & -0.233 & 0.525 & -0.259 \\
\hline & \multirow{3}{*}{ Abnormalities (\%) } & Primary & -0.404 & -0.096 & 0.239 & 0.134 & $-0.657^{\star}$ & -0.228 & -0.299 & -0.016 & -0.597 & -0.106 & 0.174 \\
\hline & & Secondary & -0.420 & 0.302 & 0.360 & -0.068 & -0.246 & 0.369 & 0.006 & 0.148 & -0.365 & 0.082 & -0.414 \\
\hline & & Tertiary & -0.161 & 0.048 & -0.015 & 0.231 & 0.097 & 0.292 & 0.400 & -0.539 & 0.032 & $0.699^{*}$ & -0.233 \\
\hline
\end{tabular}

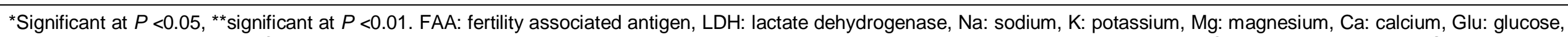

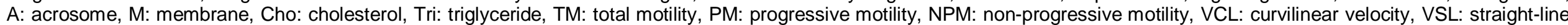
velocity, VAP: average path velocity, LIN: linearity, STR: straightness, WOB: wobble 
Lactate dehydrogenase had a positive correlation with VCL $(r=0.723, P<0.01)$ and rapid velocity $(r=$ 0.686, $P<0.05)$. Magnesium had a negative correlation with primary abnormalities $(r=-0.657)(P<0.05)$. Calcium had a positive correlation with semen volume $(r=0.663)$ and slow velocity $(r=0.639)(P<0.05)$. However, it had a negative correlation with static sperm cells $(r=-0.645)(P<0.05)$. Triglycerides had a positive correlation with normal sperm cells $(r=0.233)(P<0.01)$ and NPM $(r=0.634)(P<0.05)$. Lipids had a positive correlation with tertiary abnormalities $(r=0.699)(P<0.05)$.

\section{Discussion}

In the current study, during the evaluation of the seminal plasma constituents of unimproved indigenous and Tankwa goats, lipocalin-type prostaglandin-D synthase and osteopontin were detected. However, because they were below the detection limit of $0.5 \mathrm{mmol} / \mathrm{L}$, they could not be measured for quantity. Metalloproteinase type-2 tissue inhibitor and zinc were not detected. However, in Granadina male goats, $1.2 \pm 0.6 \mathrm{mg} / \mathrm{dL}$ of zinc was detected using the same spectrophotometry detection method and it resulted in normal semen volume and sperm cell concentration. On the other hand, the amount of zinc detected in Granadina male goats resulted in unacceptable sperm cell motility and live sperm cells (Mellado et al., 2012). In the current study, without the presence of zinc in the seminal plasma, most of the semen parameters were acceptable except sperm cell concentration.

Interestingly in this study, glucose for both breeds was higher compared with that obtained in Granadina male goats $(0.375 \mathrm{mg} / \mathrm{mL}$, converted) (Mellado et al., 2012). This shows that there are breed and environmental differences in seminal plasma constituents. Unimproved indigenous and Tankwa goats urea $(50.2 \mu \mathrm{g} / \mathrm{mL})$, cholesterol $(1.1 \mathrm{mmol} / \mathrm{L})$, calcium $(4.1 \mu \mathrm{g} / \mathrm{mL})$ and magnesium $(7.2 \mu \mathrm{g} / \mathrm{mL})$ of were lower when compared to Granadina male goats' urea $(197 \mu \mathrm{g} / \mathrm{mL})$, cholesterol $(4.5 \mathrm{mmol} / \mathrm{L})$, calcium $(108 \mu \mathrm{g} / \mathrm{mL})$ and magnesium $(19 \mu \mathrm{g} / \mathrm{mL}$ ) (converted), respectively (Mellado et al., 2012). Unimproved indigenous and Tankwa goats magnesium $(7.2 \mu \mathrm{g} / \mathrm{mL})$ and calcium $(4.1 \mu \mathrm{g} / \mathrm{mL})$ were lower when compared to the non-defined breed magnesium $(81 \mu \mathrm{g} / \mathrm{mL}$ ) and calcium $(123 \mu \mathrm{g} / \mathrm{mL}$ ) (converted), respectively (Aguiar et al., 2013). The differences between the current and previous studies might be due to environmental conditions, breeds and diets that they were fed. In Mellado et al. (2012) and Aguiar et al. (2013) studies, goats were fed a standard goat diet, whereas in the current study they grazed on natural pastures.

The levels of sodium and potassium in the current study were higher compared with the other seminal plasma constituents that were evaluated. This was expected as it was documented that their concentration is normally higher in most of the species (Colagara et al., 2009). On the other hand, protein concentration was lower in the current when compared with all the seminal plasma constituents that were evaluated. This is agreement with Mellado et al. (2012) and Aguiar et al. (2013), who reported lower protein concentration of 0.53 and $3.79 \mathrm{mmol}$ (converted), respectively. This shows that there is less demand of proteins for the survivability of sperm cells (Bozkurt et al., 2009). Although, protein levels were reported to be normally low, in the current study they were assumed to be at their peak as the study was conducted during the natural breeding season and semen quality was good (Okab, 2007). Contrary to the results of the current study, Gundouan (2006) documented that low level of protein are associated with poor semen quality. However, the physiological reasons behind this are not known.

Tankwa goats had higher VSL and VAP compared with unimproved indigenous goats. However, these were still within acceptable limit of $\geq 20 \mu \mathrm{m} / \mathrm{s}$ recommended for the sperm cells to be considered motile (Pepper-Yowell, 2011). The seminal plasma constituents obtained in the current study seem to be sufficient for unimproved indigenous and Tankwa goats, as most of the semen parameters were acceptable, except for sperm cell concentration which was below the minimum recommended level of $2 \times 10^{9}$ sperm cells $/ \mathrm{mL}$ (Hidalgo et al., 2007). Seminal plasma constituents of unimproved indigenous and Tankwa goats did not have any relationship with semen $\mathrm{pH}$. However, in species such as fish, semen $\mathrm{pH}$ showed a positive relationship with calcium, protein and cholesterol (Bozkurt et al., 2009). On the other hand, it showed a negative relationship with triglycerides and urea (Bozkurt et al., 2011). These results may mean that there are species differences in terms of seminal plasma constituents that have a relationship with semen parameters.

In unimproved indigenous goats, the fertility-associated antigen showed a positive relationship with semen volume, sperm cell concentration, TM and live sperm cells. These results are in agreement with Ax et al. (1999), who indicated that when the fertility-associated antigen is present in semen, the fertility level of the male is high. Moreover, in Tankwa goats the fertility-associated antigen showed a positive relationship with rapid velocity, VCL, VAP, sperm cell vitality and normal sperm cells. The results of the current study agree with those of the literature, as it has been indicated that proteins have a good relationship with semen quality and male fertility in other species (Ding et al., 2007; Souza et al., 2011). As a result, proteins are regarded as a valuable male fertility marker in conjunction with semen parameters in other species (Dawson et al., 2002; Dyck et al., 2011). 
In Tankwa goats, lactate dehydrogenase showed a positive relationship between TM, sperm cell vitality and viability. The results of the current study agree with those of previous studies, which documented that high levels of lactate dehydrogenase had a positive relationship with sperm cell viability, vitality (Asadpour, 2012) and motility (Ding et al., 2007). In unimproved indigenous goats, potassium showed a negative relationship with TM. These results are in line with those of Asadpour (2012), who documented that the presence of potassium is associated with decreased sperm cell motility. However, In Tankwa goats, potassium showed a positive relationship with TM, PM, and rapid and medium velocity. However, the results of the current study contradict those of Bozkurt et al. (2009), who indicated that potassium keeps the sperm cells in a motionless state. Magnesium had a negative relationship with sperm cell motility in unimproved indigenous goats. This is in line with a previous study that indicated that the presence of magnesium in the seminal plasma has a negative relationship with sperm cell motility in other species (Bozkurt et al., 2009).

In unimproved indigenous goats, calcium showed a positive relationship with TM and NPM. This is in agreement with Bozkurt et al. (2009) who reported that calcium correlated positively with sperm cell motility and negatively with sperm cell concentration in other species such as fish (Bozkurt et al., 2011). In both types of goat, calcium showed a positive relationship with TM. The results of the current study contradict those of Meseguer et al. (2004), who found that a high calcium level was associated with a lower percentage of motile sperm cells in non-defined goats. However, with overall semen parameters there were some similarities with the non-defined goats. Interestingly, the levels of calcium were higher in non-defined goats than in South African indigenous goats (Aguiar et al., 2013). The only differences were observed on the sperm cell concentration (in the previous study it was $2.5 \times 10^{9} / \mathrm{mL}$ and in the current study it was $1.5 \times$ $10^{9} / \mathrm{mL}$ ) and normal morphology (in the previous study it was $82.8 \%$ and in the current study it was $92.0 \%$ ).

The positive relationship between sperm cell motility and glucose in unimproved indigenous goats is in agreement with the study by Bozkurt et al. (2009), who indicated that the presence of glucose was connected to energy demands of the testis during spermatogenesis and lipids synthesis of sperm cells. However, that was not observed in Tankwa goats. In Tankwa goats, cholesterol showed a negative relationship with sperm cell motility parameters. The results of the current study are in line with the literature, as it has been shown that cholesterol does not have a positive relationship with sperm cell motility (Bozkurt et al., 2009) and semen volume (Meseguer et al., 2004). In contradiction, Bozkurt et al. (2009) documented that cholesterol has a protective effect against temperature changes when the semen is released, thus will result in good sperm cell characteristics.

In Tankwa goats, triglycerides showed a negative relationship with TM. The results of the current study agree with those of Bozkurt et al. (2009) who also showed a negative relationship between sperm cell motility and triglycerides. Contradictory to other studies, triglycerides showed a positive relationship with sperm cell motility (Bozkurt et al., 2011). According to Bozkurt et al. (2009), triglycerides supply energy to the sperm cells during regeneration of energy when they are not moving. Moreover, low levels of triglycerides lead to inadequate supply of energy, which leads to low sperm cell motility and fertilization capacity (Bozkurt et al., 2009). However, in the current study this was not observed because almost all the semen parameters were at acceptable standards. A positive relationship between lipids and membrane integrity in unimproved indigenous goats is in agreement with a previous study, which showed a good relationship between the two (Mandal et al., 2014). In urea in unimproved indigenous goats showed a negative relationship with semen volume, sperm cell concentration and progression. The results of the current study are in line with the literature as it has been documented that urea does not have a positive relationship with sperm cell motility and fertilizing ability (Bozkurt et al., 2009; Bozkurt et al., 2011).

\section{Conclusion}

Unimproved indigenous and Tankwa goats had similar seminal plasma constituents. The seminal plasma constituents that were detected seem to be sufficient for the sperm cells, as most of the semen characteristics were acceptable, except for sperm cell concentration. Tankwa goats had higher VSL and VAP, whereas unimproved indigenous goats had a higher level of intact acrosome. Semen parameters as measures of male fertility had significant relationships with some seminal plasma constituents. It is recommended that acceptable standards should be set for all seminal plasma constituents, as they are not currently known. Moreover, further studies need to be conducted on the seminal plasma constituents and their physiological roles.

\section{Acknowledgements}

The authors would like to thank Agricultural Research Council, Tshwane University of Technology, Department of Agriculture, Forestry and Fisheries, National Zoological Gardens of South Africa, Northern Cape Department of Agriculture, Land Reform and Rural Development, University of the Western Cape and Council for Scientific and 
Industrial Research-Southern African Science Service Centre for Climate Change and Adaptive Land Management for financial and technical contributions.

\section{Authors' Contributions}

FVR, KAN, KCL, AK, and TLN contributed on the original ideas of the manuscript. MMS and TJ contributed to data collection and interpretation. TCT gave valuable scientific inputs.

\section{Conflict of Interest Declaration}

There is no conflict of interest to declare.

\section{References}

Aguiar, G.V., Van Tilburg, M.F., Catunda, A.G.V., Celes, C.K.S., Lima, I.C.S., Campos, A.C.N., Moura, A.A.A. \& Araujo, A.A., 2013. Sperm parameters and biochemical components of goat seminal plasma in the rainy and dry seasons in the Brazilian northeast: The season's influence on the cooling of semen. Braz. J. Vet. Res. An. Sci. 65 (1), 6-12.

Asadpour, R., 2012. Relationship between mineral composition of seminal plasma and semen quality in various ram breeds. Acta. Sci. Vet. 40 (2), 1-8.

Ax, R.L., Bellin, M.E., Zhang, H.M. \& Hawkins, H.E., 1999. New approach to estimate bull fertility. In: Proceedings of The Range Beef Cow Symposium XVI.14-16 December 1999, Greeley, Colorado. http://digitalcommons.unl.edu/cgi/viewcontent.cgi?rangebeefcowsymp. (Accessed: 14.06.2015).

Bozkurt, Y., Ogretmen, F., Secer, F.S. \& Ugur, E., 2009. Relationship between seminal plasma composition and spermatological parameters in Scaly Carp (Cyprinus carpio). J. Anim. Vet. Adv. 8 (12), 2745-2749.

Bozkurt, Y., Ogretmen, F., Kokcu, O., Ugur, E., 2011. Relationships between seminal plasma composition and sperm quality parameters of the Salmo trutta macrostigma (Dumeril, 1858) semen, with emphasis on sperm motility. Czech. J. Anim. Sci. 56 (8), 355-364.

Colagara, A.H., Marzonya, E.T. \& Chaichib, M.J., 2009. Zinc levels in seminal plasma are associated with sperm quality in fertile and infertile men. Nutr. Res. 29, 82-88.

Cortada, C.N.M., Lucci, C., Gonzalez, R.A.F., Valentin, R., Mattos, C. B., 2000. Plasma urea levels on reproductive parameters of wool-less rams (Ovis Aries, LINNAEUS, 1758). Braz. J. Vet. Res. An. Sci. 37 (6), 87-91.

Cross, N.L. 1998. Role of cholesterol in sperm capacitation. Biol. Reprod. 59, 7-11.

Dawson, G.R., Oyarzo, J.N., Bellin, M.E., Zhang, H.M., McCauley, T.C. \& Ax, R.L., 2002. Promising new technologies for semen evaluation. In: Proceedings of the applied reproductive strategies in beef cattle workshop, 5-6 September 200 in Manhattan, Kansas. http:beefrepro.unl.edu/proceedings/2002manhattan/13ksunewtechax.pdf (Accessed: 12.02.2013).

Ding, Z., Qu, F., Guo, W., Ying, X., Wu, M. \& Zhang, Y., 2007. Identification of sperm forward motility-related proteins in human seminal plasma. Mol. Reprod. Dev. 74, 1124-1131.

Dyck, M.K., Foxcroft, G.R., Novak, S., Ruiz-Sanchez, A., Patterson, J. \& Dixon, W.T., 2011. Biological markers of boar fertility. Reprod. Domest. Anim. 46, 55-58.

Gundouan, M., 2006. Some reproductive parameters and seminal plasma constituents in relation to season in Akkaraman and Awassi rams. Turk. J. Vet. Anim. Sci. 30, 95-100.

Hidalgo, M., Rodriguez, I. \& Dorado, J., 2007. The effect of cryopreservation on sperm head morphometry in Florida male goat related to sperm freezability. Anim. Reprod. Sci. 100, 61-72.

Jonker, T., 2013. Tankwa feral goats - A real cliff hanger or the end of the road. Carnarvon Research Station, 5 February 2013. Electronic copy.

Kasimanickam, R., 2011. Application of technology in male reproduction. In: Proceedings of Applied Reproductive Strategies in Beef Cattle, 30 September-1 October, 2011, Northwest, Bois. http:beefrepro.unl.edu/ beefrepro.unl.edu/proceedings/2011northwest/2011NorthwestARSBC.pdf. (Accessed: 12.02.2013).

Klein, T., Cooper, T.G. \& Yeung, C.H., 2006. The role of potassium chloride cotransporters in murine and human sperm volume regulation. Biol. Reprod. 75, 853-858.

Kotze, A., Grobler, J.P., Van Marle-Koster, E., Jonker, T. \& Dalton, D.L., 2014. The Tankwa Karoo National Park feral goat population: A unique genetic resource. S. Afr. J. Anim. Sci. 44, 43-48.

Loskutoff, N.M. \& Crichton, E.G., 2001. Standard operating procedures for genome resource banking. The Bill and Bernience Grewcock Center for Conservation and Research, Omaha's Henry Doorly Zoo.1-16.

Mandal, R., Badyakar, D. \& Chakrabarty, J., 2014. Role of membrane lipids fatty acids in sperm cryopreservation. Adv. Androl, 1-9.

Manzi, M., Rutagwenda, T., Kanuya, N. \& Chatikobo, P., 2011. Phenotypic characterization of goats raised under traditional husbandry systems in Bugesera and Nyagatare districts of Rwanda. J. Anim. Vet. Adv. 10 (24), 3297-3302.

Massanyi, P., Weis, J., Lukac, N., Trandzik, J. \& Bystricka, J., 2008. Cadmium, zinc, copper, sodium and potassium concentrations in rooster and turkey semen and their correlation. J. Environ. Sci. Health. 43, 563-565.

Matshaba, B., 2010. Characterisation and cryopreservation of South African unimproved indigenous goat semen. MSc dissertation, Bloemfontein, University of the Free State, South Africa.

Mellado, M., Herrera, C.A.M., Arevalo, J.R., Garcia, J.E. \& Veliz, F.G., 2012. Effect of dietary energy intake and somatotropin administration after weaning on growth rate and semen characteristics of Granadina goat bucks. Turk. J. Vet. Anim. Sci. 36 (4), 338-345. 
Meseguer, M., Garrido, N., Martinez-Conejero, J.A., Simon, C., Pellicer, A. \& Remohi, J., 2004. Relationship between standard semen parameters, calcium, cholesterol contents, and mitochondrial activity in ejaculated spermatozoa from fertile and infertile males. J. Assist. Reprod. Genet. 21 (12), 445-451.

Naing, S.W., Wahid, H., Azam, K.M, Rosnins, Y., Zuki, A.B., Kazhal, S., Bukar, M.M., Thein, M., Kyaw, T. \& San, M.M., 2010. Effects of sugar on characteristics of Boer goat semen after cryopreservation. Anim. Reprod. Sci. 122, 23-28.

Naing, S.W., Haron, A.W., Goriman, M.A.K., Yusoff, R., Bakar, M.Z.A., Sarsaifi, K., Bukar, M.M., Thein, M., Kyaw, T. \& San, M.M., 2011. Effect of seminal plasma removal, washing solutions, and centrifugation regimes on Boer goat semen cryopreservation. Pertanika J. Trop. Agric. 34 (2), 271-279.

Nikoobakht, M., Aloosh, M. \& Hasani, M., 2005. Seminal plasma magnesium and premature ejaculation: A case-control study. J. Uro. 2 (2), 102-105.

Okab, O.B., 2007. Semen characteristics and plasma testosterone of New Zealand male rabbits as affected by environmental temperatures. Slovak. J. Anim. Sci. 40 (4), 161-167.

Pepper-Yowell, A.R., 2011. The use of computer assisted semen analysis to predict fertility in Holstein bulls. Msc. dissertation. Colorado, Colorado State University.

Purdy, P.H., 2006. A review on goat sperm cryopreservation. Small Rumin. Res. 63, 215-225.

Ramsay, K.A. \& Donkin, E.F., 2000. A review of the current status of goat research and development in South Africa. Proceedings of Regional workshop on goat development in Southern Africa, 31 July-4 August 2000, Malawi, Mangochi. www.researchgate.net/.../237254983areviewofthecurrents...pdf. (Accessed: 13.04.2014).

Samper, C.J., 2000. Equine breeding management and artificial insemination. Second edition. Elsevier Saunder, USA.

Sorensen, M.B., Bergdahl, I.A., Hjollund, N.H.I., Bonde, J.P.E., Stoltenberg, M. \& Ernst, E., 1999. Zinc, magnesium and calcium in human seminal fluid: Relations to other semen parameters and fertility. Mol. Hum. Reprod. 5 (4), 331-337.

Souza, C.E.A., Moura, A.A., Lima-Souza, A.C. \& Killian, G.J., 2011. Binding patterns of seminal plasma proteins on bovine epididymal and ejaculated sperm membrane. Braz. J. Vet. Res. Anim. Sci. 63 (3), 535-543.

Teixeira, A.V.C., Eloy, A.M.X., Furtado, J.R., Pinheiro, R.R. \& Ponte, M.S., 2009. 1D mapping of seminal plasma proteins in Anglo-Nubian goats. Anim. Reprod. 6, 516-525.

Webb, E.C., Cronje, P.B. \& Donkin, E.F. (ed.), 1998. Research and training strategies for goat production systems in South Africa. In: Proceedings of Goat Production in South Africa: Constraints and Opportunities Workshop, 22-26 November 1998, Eastern Cape, South Africa. http://www.ais.up.ac.za/vet/goat/documents/hogsproceed.pdf. (Accessed: 12.08.2012).

Yakubu, A., Raji, A.O. \& Omeje, J.N., 2010. Genetic and phenotypic differentiation of qualitative traits in Nigerian indigenous goat and sheep populations. Asian Research Publishing Network 5 (2), 58-66.

Yami, A. \& Merkel, R.C. (ed.), 2008. Sheep and goat production handbook for Ethiopia: Ethiopia Sheep and Goat Productivity Improvement Program (ESGPIP). Branna Printing Enterprise, Ethiopia.

Yeung, C.H. \& Cooper, T.G., 2008. Potassium channels involved in human sperm volume regulation-quantitative studies at the protein and mRNA levels. Mol. Reprod. Dev. 75, 659-668. 